\title{
Successful Treatment of Vaccine-Induced Immune Thrombotic Thrombocytopenia in a 26-Year-Old Female Patient
}

\author{
Marcel Kemper Georg Lenz Rolf Michael Mesters \\ Department of Medicine A - Hematology, Oncology, Hemostaseology and Pneumology, University Hospital \\ Münster, Münster, Germany
}

\section{Keywords}

Vaccine-induced immune thrombotic thrombocytopenia •

Thrombocytopenia · Thrombosis · Vaccination · Severe acute respiratory syndrome coronavirus 2

\begin{abstract}
Vaccine-induced immune thrombotic thrombocytopenia (VITT) has already been described after vaccination with ChAdOx2 nCov-19 (AstraZeneca) and Ad26.COV2.S (Johnson \& Johnson/Janssen). However, less knowledge so far has been gained about optimal therapeutic regimens in VITTsuspected patients. Here, we report the case of a 26-year-old female patient, who developed bilateral deep vein thrombosis in the lower legs and severe thrombocytopenia after ChAdOx2 nCov-19 vaccination. After initial anticoagulation therapy regimens including fondaparinux, apixaban, and danaparoid failed, the patient was successfully treated with high-dose intravenous immunoglobulins in combination with parental anticoagulation therapy with argatroban. As vaccination against severe acute respiratory syndrome coronavirus 2 affects billions of people worldwide, medical facilities and hospitals have to be prepared and provide effective treatment options in VITT-suspected patients, including rapid application of high-dose intravenous immunoglobulins, to improve patient outcomes.

(c) 2021 S. Karger AG, Basel
\end{abstract}

\section{Introduction}

Vaccine-induced immune thrombotic thrombocytopenia (VITT) has evolved as a new term for thrombosis associated with thrombocytopenia after vaccination with the adenoviral vector vaccine ChAdOx1nCov-19 (AstraZeneca) against severe acute respiratory syndrome coronavirus 2 (SARS-CoV-2). These thrombotic events are characterized by their predominant location in cerebral veins and high risk of adverse or even fatal outcome [1, 2]. Despite recent achievements in understanding the underlying mechanisms $[1,2]$, there is still a lack of knowledge especially with regard to the optimal treatment approach. Here, we report a case of a 26-year-old female patient who developed bilateral deep vein thrombosis (DVT) in the lower legs and thrombocytopenia after vaccination with ChAdOx1nCov-19, who was successfully treated with the combination of high-dose intravenous immunoglobulins (IVIG) and anticoagulation with intravenous argatroban followed by oral rivaroxaban.

\section{Case Report}

A 26-year-old female patient was vaccinated with the 1st dose of ChAdOx1nCov-19 on March 26, 2021. The patient was taking a progestogen-only oral contraceptive (desogestrel $75 \mu \mathrm{g}$ ) for 1 karger@karger.com

www.karger.com/aha

(C) 2021 S. Karger AG, Base

Karger"
Correspondence to:

Marcel Kemper, marcel.kemper@ukmuenster.de 


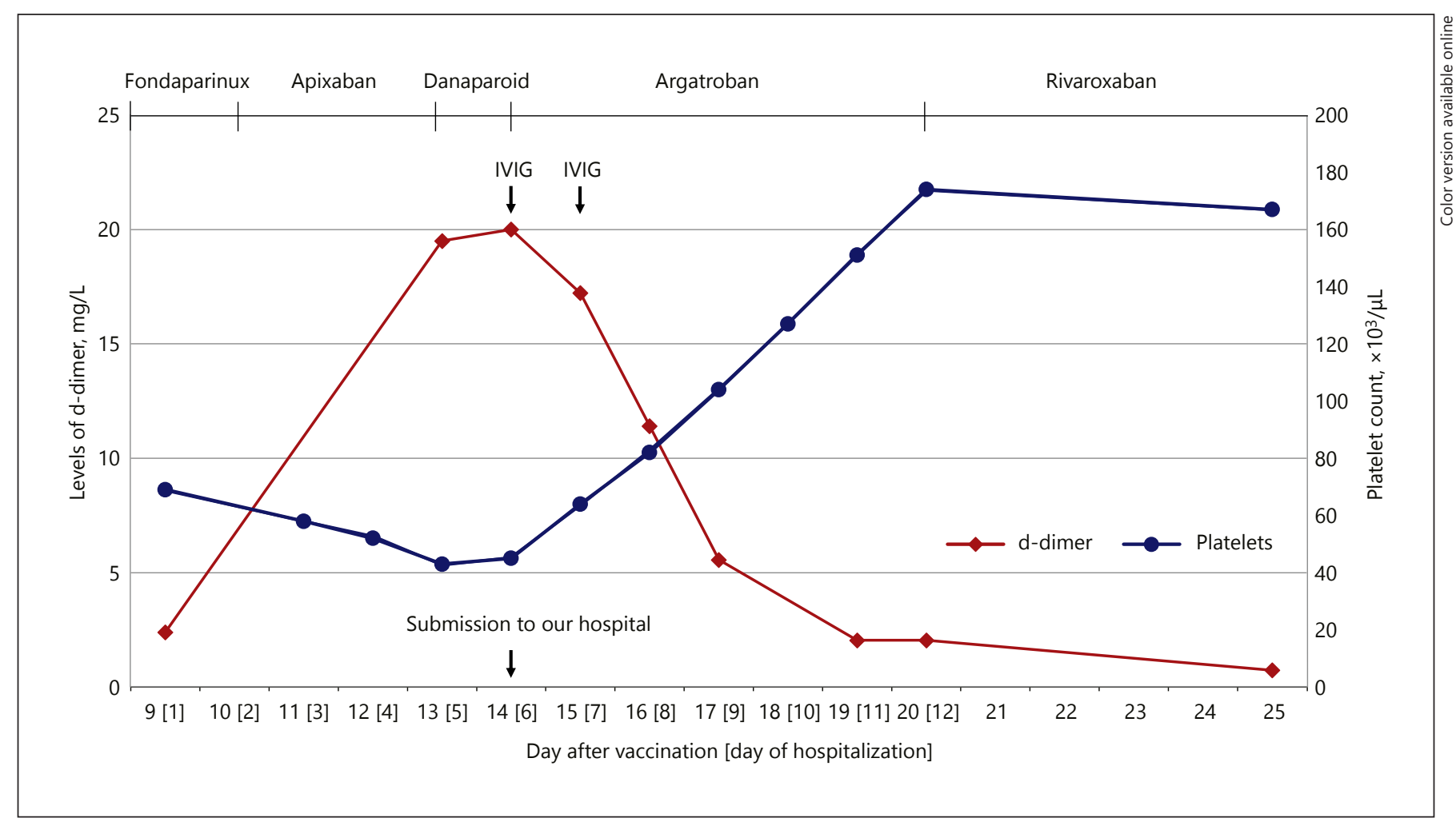

Fig. 1. Levels of D-dimer and platelet count under treatment. After application of high-dose IVIG ( $1 \mathrm{~g} / \mathrm{kg}$ on days 1 and 2) and treatment with i.v. argatroban, levels of D-dimer slowly decreased (red line) and platelet counts increased (blue line). IVIG, intravenous immunoglobulin.

year and was obese $\left(\mathrm{BMI} 31 \mathrm{~kg} / \mathrm{m}^{2}\right)$ but a nonsmoker. There were no other thrombotic risk factors such as immobilization, previous hospital treatment, or surgical procedure, and there was no history of heparin exposure. The only co-medication was atenolol due to mild arterial hypertension. Nine days after vaccination, the patient was suffering from pain and swelling of the right lower leg. A blood test showed elevated D-dimer levels $(2.39 \mathrm{mg} / \mathrm{L}$ Fibrinogen Equivalent Units [FEU]; upper limit of normal $<0.5 \mathrm{mg} / \mathrm{L} \mathrm{FEU}$ ) and isolated thrombocytopenia $(69,000 / \mu \mathrm{L})$. Pseudothrombocytopenia was excluded. Venous ultrasound revealed a DVT in the right popliteal vein. Investigation of antibodies against the PF4heparin/complex was not available at the time of diagnosis. After initial therapeutic anticoagulation with s.c. fondaparinux $(7.5 \mathrm{mg}$ once daily), anticoagulation was switched to apixaban because of worsening thrombocytopenia $(43,000 / \mu \mathrm{L}$; Fig. 1). Venous ultrasound was repeated on day 5 of hospitalization (day 13 after vaccination) and found progressive thrombosis with bilateral DVT in the posterior tibial and fibular veins. Clinically at this time, there were no symptoms of cerebral vein thrombosis (CVT) or pulmonary embolism. Echocardiography showed no right ventricular dilatation, and abdominal ultrasound revealed no splanchnic-vein thrombosis. As the results for antibodies against PF4/heparin complexes were available and eventually resulted positive (Asserachrom ${ }^{\circledR}$ HPIA-IgG, Stago), therapy was subsequently switched to danaparoid. Meanwhile, the patient was assigned to our Univer- sity Hospital for further diagnostic evaluation and treatment. Because of persistent high D-dimer levels ( $>20 \mathrm{mg} / \mathrm{L} \mathrm{FEU}$ ) and thrombocytopenia despite anticoagulation with danaparoid (Fig. 1), we immediately switched therapy to the intravenous thrombin inhibitor argatroban with a target APTT of $80-100 \mathrm{~s}$ (2.5-3.0 the upper limit of the normal range) combined with highdose IVIG $(1 \mathrm{~g} / \mathrm{kg})$ on days 1 and 2 after submission. As the patient subsequently complained of headache, we performed cerebral magnetic tomography imaging (MRI), which excluded CVT. Slowly, D-dimer levels decreased and platelet counts increased during the course of therapy with argatroban and IVIG application (Fig. 1). As the patient developed a more severe headache and started vomiting, cerebral contrast-enhanced MRI was repeated, and CVT was again excluded. Venous ultrasound control on day 9 of hospitalization (day 17 after vaccination) revealed residual thrombotic material in the right fibular vein. Plasma samples for the heparin-induced platelet-activation assay taken before IVIG application and sent to an external specialized laboratory (Medilys, Hamburg, Germany) showed a negative test result. After laboratory (platelets $>150,000 / \mu \mathrm{L}$; D-dimer $2.04 \mathrm{mg} / \mathrm{L}$ ) and clinical recovery, we switched the anticoagulation regimen from argatroban to oral rivaroxaban ( $15 \mathrm{mg}$ BID for 21 days followed by $20 \mathrm{mg}$ OD) and discharged the patient from our hospital. The clinical and laboratory follow-up was uneventful in the outpatient setting. 


\section{Discussion/Conclusion}

When researchers found antibody formation against PF4-polyanion complexes followed by platelet activation to be the underlying mechanism of VITT, one of the first proposed treatment strategies was to block the interaction via the Fcy receptor by administration of high-dose IVIG $[1,2]$. Furthermore, the authors also suggested a rather complex diagnostic management scheme for patients suspected with VITT [1].

In Germany so far (up until April 30, 2021), 67 cases of thrombotic thrombocytopenia after almost 5.8 million vaccinations with ChAdOx1nCov-19 have been reported (corresponding to an absolute risk of 1:87,000 with a high case fatality rate of 21\%) [3]. Similar numbers are reported from the United Kingdom (UK) with 242 cases of thrombotic thrombocytopenia after almost 23 million vaccinations up till April 28th (absolute risk of 1:95,000 with a high case fatality rate of 20\%) [4]. This might indicate that the rather time-consuming diagnostic pathway, which is suggested by some authors, is problematic when there is need for urgent decision-making because results for heparin/PF4 antibody or heparin-induced plateletactivation assay testing are not readily available in the vast majority of hospitals. In contrast, another suggested diagnostic pathway that is guided by rapidly available laboratory tests (blood count and D-dimer level) appears more suitable for daily clinical routine [5].

In this case report, we confirm that immediate IVIG application in combination with parental anticoagulation therapy with intravenous argatroban is an effective treatment option for patients with suspected VITT. These data also indicate that previous therapeutic regimens in this young woman (fondaparinux and apixaban) without IVIG administration may not be sufficient in VITT as demonstrated by progressive thrombosis and persistent high D-dimer levels (>20 mg/L FEU). However, as VITT is recognized as a new clinical syndrome, there are little published data about effective treatment options apart from single or serial case reports. Although IVIG and argatroban were effective in this young woman, this regimen might not be successful in all patients with VITT, particularly in those with CVT and refractory thrombocytopenia. Therapeutic plasma exchange has been shown to be effective in some of these patients with refractory thrombocytopenia after IVIG application [6]. Alternative immunosuppressive therapies such as glucocorticoids and rituximab are also discussed in the literature $[5,6]$, and endovascular treatment can be considered in patients with extensive CVT $[7,8]$, but the role of those treatments remains unclear. Further studies are needed to investigate and compare different treatment strategies in patients with VITT.

So far, no risk factors for development of this severe complication, named VITT, have been identified. Especially, the role of classic "thrombophilic" risk factors (e.g., inherited or acquired thrombophilia, combined oral contraceptives, hormone replacement therapy, recent surgery, smoking, and obesity) is unclear. It is well known that the incidence of venous thromboembolism (VTE), especially CVT, in "non-VITT patients" is higher in females of reproductive age, with about $50 \%$ of these cases carrying an acquired procoagulant risk factor (e.g., combined oral contraception, hormone replacement therapy, pregnancy, or puerperium) $[9,10]$. However, the patient in this case report took the progestogen-only contraceptive desogestrel $75 \mu \mathrm{g}$ OD which is not associated with a significant increased risk for VTE [11] in contrast to combined oral contraceptives $[12,13]$. Thus, it may not be suitable to compare common cases of CVT with VITTassociated CVT cases, as the underlying mechanisms and risk factors might differ. Although in Germany $75 \%$ of the patients with VITT after ChAdOx1 nCov-19 vaccination were women (50 out of 67) and only 12 of them were older than 60 years [3], the numbers are too low to conclude that VITT predominantly affects younger women especially when considering that the vaccination campaign (regarding the ChAdOx1 nCov-19 vaccine) in Germany initially was focused on younger people below 60 years of age. In contrast, only $58 \%$ (141 out of 242) of patients with VITT after vaccination with ChAdOx1 nCov19 in the UK were female [4], indicating a relevant impact of the country's vaccination campaign on gender differences in incidence numbers. Thus, when a cutoff value at the age of 60 is used to allocate ChAdOx1nCov-19 among people, we need to remain aware of VITT in older and male people.

In our patient, no thrombophilic risk factors were identified (e.g., antithrombin, protein C or S deficiency, Factor V Leiden or prothrombin mutation [G20210A], and antiphospholipid antibodies), and the patient was in a healthy condition before onset of symptoms, which is in line with previous findings in VITT patients $[1,2,5]$. This supports the notion that VITT patients cannot be identified, so far, by common inherited or acquired risk factors. Due to the global SARS-CoV-2 pandemic, billions of people will be vaccinated eventually. Hence, we have to be prepared as VITT will most likely occur in thousands of people. In order to better understand this clinical syndrome and to improve patient outcomes, it is important 
to share clinical features, patient characteristics, and possible effective treatment strategies of VITT cases amongst the medical community.

In conclusion, we recommend applying IVIG and parental therapeutic anticoagulation with a nonheparin anticoagulant like argatroban i.v. as soon as possible in all patients with VTE when there is a high degree of suspicion for VITT. This strategy probably improves patient outcomes significantly. After reaching a clinical and laboratory recovery with platelets $>150,000 / \mu \mathrm{L}$ and confirmation of VITT by laboratory tests, anticoagulation therapy can then be switched from argatroban to a direct oral anticoagulant such as rivaroxaban. However, alternative treatment options (such as therapeutic plasma exchange, glucocorticoids, and rituximab) should be considered in patients with refractory thrombocytopenia after IVIG application.

\section{Statement of Ethics}

Ethical approval was not required, as the patient in this case report was not involved in any study. Written informed consent was obtained from the patient for publication of this case report and any accompanying data and images.

\section{Conflict of Interest Statement}

M.K. declares no conflicts of interest. G.L. has received honoraria from AstraZeneca and Janssen, has participated in a consulting or advisory role for AstraZeneca and Janssen, has been on a speaker's bureau for AstraZeneca and Janssen, and has received research funding from AstraZeneca and Janssen. R.M.M. has received honoraria from Bayer HealthCare, Daiichi-Sankyo, and Pfizer.

\section{Funding Sources}

There was no funding for this case report.

\section{Author Contributions}

M.K. wrote the manuscript. G.L. and R.M.M. revised the manuscript. All authors read and approved the final manuscript.

\section{Data Availability Statement}

All data generated or analyzed are included in this case report. Further enquiries can be directed to the corresponding author.

\section{References}

1 Greinacher A, Thiele T, Warkentin TE, Weisser K, Kyrle PA, Eichinger S. Thrombotic thrombocytopenia after ChAdOx1 nCov19 vaccination. N Engl J Med. 2021;384(22): 2092-101.

2 Schultz NH, Sørvoll IH, Michelsen AE, Munthe LA, Lund-Johansen F, Ahlen MT, et al. Thrombosis and thrombocytopenia after ChAdOx1 nCoV-19 vaccination. N Engl J Med. 2021;384(22):2124-30.

3 Bundesinstitut für Impfstoffe und biomedizinische Arzneimittel (Paul-Ehrlich-Institut). Sicherheitsbericht: Verdachtsfälle von Nebenwirkungen und Impfkomplikationen nach Impfung zum Schutz vor COVID-19. 07.05.2021.

4 Medicines \& Healthcare products Regulatory Agency (MHRA). Research and analysis, coronavirus vaccine - weekly summary of yellow card reporting. 2021 [updated 6 May 2021].
5 Scully M, Singh D, Lown R, Poles A, Solomon $\mathrm{T}$, Levi $\mathrm{M}$, et al. Pathologic antibodies to platelet factor 4 after ChAdOx $1 \mathrm{nCoV}-19 \mathrm{vac}-$ cination. N Engl J Med. 2021;384(23):220211.

6 Patriquin CJ, Laroche V, Selby R, Pendergrast J, Barth D, Côté B, et al. Therapeutic plasma exchange in vaccine-induced immune thrombotic thrombocytopenia. N Engl J Med. 2021; 385(9):857-9.

7 Ropper AH, Klein JP. Cerebral venous thrombosis. N Engl J Med. 2021;385(1):59-64.

8 Coutinho JM, Zuurbier SM, Bousser MG, Ji X, Canhão P, Roos YB, et al. Effect of endovascular treatment with medical management vs standard care on severe cerebral venous thrombosis: the TO-ACT randomized clinical trial. JAMA Neurol. 2020;77(8):966-73.

9 Coutinho JM, Zuurbier SM, Aramideh M, Stam J. The incidence of cerebral venous thrombosis: a cross-sectional study. Stroke. 2012;43(12):3375-7.
10 Devasagayam S, Wyatt B, Leyden J, Kleinig T. Cerebral venous sinus thrombosis incidence is higher than previously thought: a retrospective population-based study. Stroke. 2016;47(9):2180-2.

11 Tepper NK, Whiteman MK, Marchbanks PA, James AH, Curtis KM. Progestin-only contraception and thromboembolism: a systematic review. Contraception. 2016;94(6):678-700.

12 de Bastos M, Stegeman BH, Rosendaal FR, Van Hickam Vlieg A, Helmerhorst FM, Stijnen $\mathrm{T}$, et al. Combined oral contraceptives: venous thrombosis. Cochrane Database Syst Rev. 2014;3:CD010813.

13 Dragoman MV, Tepper NK, Fu R, Curtis KM, Chou R, Gaffield ME. A systematic review and meta-analysis of venous thrombosis risk among users of combined oral contraception. Int J Gynaecol Obstet. 2018;141(3):287-94. 